PROCEEDINGS OF THE

AMERICAN MATHEMATICAL SOCIETY

Volume 137, Number 7, July 2009, Pages 2375-2385

S 0002-9939(09)09781-0

Article electronically published on January 29, 2009

\title{
POINTWISE APPROXIMATION BY ELEMENTARY COMPLETE CONTRACTIONS
}

\author{
BOJAN MAGAJNA \\ (Communicated by Marius Junge)
}

\begin{abstract}
A complete contraction on a $C^{*}$-algebra $A$, which preserves all closed two sided ideals $J$, can be approximated pointwise by elementary complete contractions if and only if the induced map on $B \otimes A / J$ is contractive for every $C^{*}$-algebra $B$, ideal $J$ in $A$ and $C^{*}$-tensor norm on $B \otimes A / J$. A lifting obstruction for such an approximation is also obtained.
\end{abstract}

\section{INTRODUCTION AND NOTATION}

We often try to understand the structure of operators and spaces on which they act in terms of approximation by finite rank maps. On a $C^{*}$-algebra $A$, however, we may regard two-sided multiplications $x \mapsto a x b$ as basic building blocks (instead of rank one operators). We can try to approximate a more general map $\phi$ on $A$, one that preserves ideals, by finite sums of two-sided multiplications, that is, by elementary operators. Here by an ideal we will always mean a closed two-sided ideal, and we denote the set of all such ideals in $A$ by $\mathcal{I}_{\mathrm{c}}(A)$.

The class of separable $C^{*}$-algebras $A$ on which every (completely) bounded map preserving ideals admits uniform approximation by elementary operators turns out to be very restrictive: it includes just finite direct sums of $C^{*}$-algebras of continuous sections vanishing at $\infty$ of locally trivial $C^{*}$-bundles of finite type [15. On the other hand, each bounded linear map on an arbitrary $C^{*}$-algebra $A$ that preserves ideals can be approximated pointwise by elementary operators [12, 1 .

In this paper we study the question of pointwise approximation by elementary complete contractions, that is, by completely contractive elementary operators. It turns out that the uniform bound on the norms of approximating operators has a strong effect on the nature of the problem. We note (although this will not be used here) that for a complete contraction $\phi$ on a $C^{*}$-algebra $A$ that preserves ideals and for a fixed $x \in A$ there will always exist a net of elementary complete contractions $\phi_{k}$ on $A$ such that $\left\|\phi(x)-\psi_{k}(x)\right\|$ tends to 0 [13. However, given a finite collection $x_{1}, \ldots, x_{n}$ of elements, there is in general no common approximating net for all $x_{j}$ simultaneously. This problem turns out to be closely related to the theory of $C^{*}$-tensor norms.

Received by the editors October 18, 2007, and, in revised form, September 27, 2008.

2000 Mathematics Subject Classification. Primary 46L06, 46L07; Secondary 47B47.

Key words and phrases. $C^{*}$-algebra, $C^{*}$-tensor products, ideals, elementary operators, point norm topology. 
In the case of a von Neumann algebra $R$, it can be deduced from results of Chatterjee and Smith [6] that each complete contraction on $R$ which preserves weak* closed two-sided ideals can be approximated by elementary complete contractions in the point weak* topology if and only if $R$ is injective (at least if $R$ has a separable predual). When trying to apply this result to study the approximation on general $C^{*}$-algebras (via the universal von Neumann envelopes), we have encountered difficulties, since $C^{*}$-algebras are not in general locally reflexive as operator spaces.

A motivation for this paper was the desire to understand the range (and its completions in various topologies) of the natural map $\mu$ from the Haagerup tensor product $A \stackrel{h}{\otimes} A$ (or $M(A) \stackrel{h}{\otimes} M(A))$ into $\mathrm{CB}(A)$. This is a complementary problem to the question of when $\mu$ is isometric, which has been solved by Somerset 20] and by Archbold, Timoney and Somerset [2]. Important special cases were studied earlier by many others (see [19, [1] and the references therein).

In Section 2 we obtain a necessary and sufficient condition for a map $\phi$ on a $C^{*}$-algebra $A$ to be pointwise approximable by elementary complete contractions. Besides the obvious requirement that $\phi(J) \subseteq J$ for each ideal $J$ of $A$, it is also necessary that the induced map on $B \otimes A / J$ be contractive for every $C^{*}$-tensor norm on the algebraic tensor product of $A / J$ with each $C^{*}$-algebra $B$. We prove that these conditions are also sufficient, relate them to representations of $A$, derive some consequences and present a concrete example. It follows in particular that every nuclear $C^{*}$-algebra has the elementary completely contractive approximation property (the ECCAP, for short) in the sense that all complete contractions that preserve ideals can be pointwise approximated by elementary ones. The author does not know if the converse is true.

It is not always easy to verify the conditions of our basic result (Theorem 2.1 below), since often we do not possess enough knowledge about all possible $C^{*}$-tensor norms concerned. Therefore we derive in Section 3 another necessary condition for approximation by elementary complete contractions, based on the possibility of lifting. As an example, we apply this, together with Ozawa's characterization of the local lifting property [16], to show that the Calkin algebra does not have the ECCAP.

It is customary to take the coefficients $a_{i}$ and $b_{i}$ of an elementary operator $\psi(x)=$ $\sum_{i=1}^{n} a_{i} x b_{i} \quad(x \in A)$ to be in the multiplier algebra $M(A)$ of $A$, but, when studying the pointwise approximation, we can use an approximate unit of $A$ to modify the coefficients to be in $A$. The set of all elementary operators on $A$ (with coefficients in $A$ ) is denoted by $\mathrm{E}(A)$ and the unit ball in $\mathrm{E}(A)$ (in the completely bounded norm) by $\mathrm{E}_{1}(A)$. By $\operatorname{ICB}(A)$ we shall denote all completely bounded maps that preserve all closed ideals in $A$ and by $\operatorname{ICB}_{1}(A)$ the unit ball of $\operatorname{ICB}(A)$. The closure

of a set $S$ will be denoted by $\overline{\bar{S}}$ for the norm topology, by $\bar{S}^{\text {p.n. }}$ for the point norm topology, and by $\bar{S}$ for the weak* topology.

For basic facts concerning completely bounded maps, operator spaces and tensor norms, we refer to any of the books [4], [8], [17, [18], 22].

\section{Approximation by elementary operators and $C^{*}$-Tensor nORMS}

Theorem 2.1. The following three statements are equivalent for a complete contraction $\phi$ on a $C^{*}$-algebra $A$ : 
(i) $\phi \in{\overline{\mathrm{E}_{1}(A)}}^{\text {p.n. }}$.

(ii) For each representation $\pi: A \rightarrow \mathrm{B}(\mathcal{H})$ and all finite subsets $\left\{a_{1}, \ldots, a_{n}\right\}$ of $A$ and $\left\{a_{1}^{\prime}, \ldots, a_{n}^{\prime}\right\}$ of $\pi(A)^{\prime}$ the following inequality holds:

$$
\left\|\sum_{i=1}^{n} a_{i}^{\prime} \pi\left(\phi\left(a_{i}\right)\right)\right\| \leq\left\|\sum_{i=1}^{n} a_{i}^{\prime} \pi\left(a_{i}\right)\right\| .
$$

(iii) The map $\phi$ preserves all ideals $J \in \mathcal{I}_{\mathrm{c}}(A)$, and for each $C^{*}$-algebra $B$ and $C^{*}$-tensor norm $\|\cdot\|$ on $B \otimes A / J$ the map $1 \otimes \phi_{J}$ on $B \otimes A / J$ (where $\phi_{J}$ on $A / J$ is defined by $\left.\phi_{J}(a+J)=\phi(a)+J\right)$ is a contraction.

Proof. Clearly (i) implies (ii). To prove the implication (ii) $\Rightarrow$ (iii), first note that $\phi$ preserves each ideal $J$ of $A$ (take in (2.1) $n=1, a_{1}^{\prime}=1$ and $\pi$ such that $\operatorname{ker} \pi=J$ ). Given a $C^{*}$-algebra $B$ and a tensor norm $\|\cdot\|$ on $B \otimes A / J$ as in (iii), we first extend the tensor norm to $\tilde{B} \otimes \tilde{A} / J$, where $\tilde{B}$ is the unitization of $B$ (if $B$ is not unital, and $\tilde{B}=B$ if $B$ is unital; see [21, IV.4.3]). Let $\Psi$ be a faithful representation of the completion of $\tilde{B} \otimes \tilde{A} / J$ on a Hilbert space $\mathcal{H}, q: \tilde{A} \rightarrow \tilde{A} / J$ the quotient map, and define $\pi: A \rightarrow \mathrm{B}(\mathcal{H})$ by

$$
\pi(a)=\Psi(1 \otimes q(a)) .
$$

Given $w=\sum_{i=1}^{n} b_{i} \otimes q\left(a_{i}\right)$ in $\tilde{B} \otimes \tilde{A} / J$, we put $a_{i}^{\prime}=\Psi\left(b_{i} \otimes 1\right)$. Then $a_{i}^{\prime}$ commutes with $\Psi(1 \otimes A / J)=\pi(A)$. Using (2.1) and the fact that $\Psi$ is isometric we have that

$$
\begin{aligned}
\left\|\left(1 \otimes \phi_{J}\right)(w)\right\| & =\left\|\Psi\left(\sum_{i=1}^{n} b_{i} \otimes q\left(\phi\left(a_{i}\right)\right)\right)\right\| \\
& =\left\|\sum_{i=1}^{n} \Psi\left(b_{i} \otimes 1\right) \Psi\left(1 \otimes q\left(\phi\left(a_{i}\right)\right)\right)\right\| \\
& =\left\|\sum_{i=1}^{n} a_{i}^{\prime} \pi\left(\phi\left(a_{i}\right)\right)\right\| \\
& \leq\left\|\sum_{i=1}^{n} a_{i}^{\prime} \pi\left(a_{i}\right)\right\| \\
& =\left\|\sum_{i=1}^{n} \Psi\left(b_{i} \otimes 1\right) \Psi\left(1 \otimes q\left(a_{i}\right)\right)\right\| \\
& =\left\|\Psi\left(\sum_{i=1}^{n} b_{i} \otimes q\left(a_{i}\right)\right)\right\| \\
& =\|w\| .
\end{aligned}
$$

Now we will prove the implication (ii) $\Rightarrow$ (i). If $\phi \notin{\overline{\mathrm{E}_{1}(A)}}^{\text {p.n. }}$, then for some $n \in \mathbb{N}$ and $a=\left(a_{1}, \ldots, a_{n}\right) \in A^{n}$ the element $y:=\left(\phi\left(a_{1}\right), \ldots, \phi\left(a_{n}\right)\right)$ is not contained in the norm closure of the subset

$$
S:=\left\{\left(\psi\left(a_{1}\right), \ldots, \psi\left(a_{n}\right)\right): \psi \in \mathrm{E}_{1}(A)\right\}
$$

of $\tilde{A}^{n}$ (with the max norm). By [14, 1.1] there exist a representation $\pi: \tilde{A} \rightarrow \mathrm{B}(\mathcal{H})$ (for some Hilbert space $\mathcal{H}$, so that $\mathrm{B}(\mathcal{H})$ is an $\tilde{A}$-bimodule via $\pi$ ) and a completely bounded $\tilde{A}$-bimodule map $\Phi: \tilde{A}^{n} \rightarrow \mathrm{B}(\mathcal{H})$ such that

$$
\|\Phi(x)\| \leq 1 \text { for all } x \in S \text { and }\|\Phi(y)\|>1 .
$$

The elements $a_{i}^{\prime}:=\Phi\left(e_{i}\right)$, where $e_{i}=(0, \ldots, 1, \ldots, 0)$, are in $\pi(\tilde{A})^{\prime}$ since for all $a \in \tilde{A}$,

$$
a_{i}^{\prime} \pi(a)=\Phi\left(e_{i}\right) \pi(a)=\Phi\left(e_{i} a\right)=\Phi\left(a e_{i}\right)=\pi(a) \Phi\left(e_{i}\right)=\pi(a) a_{i}^{\prime} .
$$

Further, since $\Phi$ is an $A$-bimodule map, $\Phi\left(x_{1}, \ldots, x_{n}\right)=\sum a_{i}^{\prime} \pi\left(x_{i}\right)$ for all $x_{i} \in A$, and hence (2.2) can be rewritten as

$$
\left\|\sum_{i=1}^{n} a_{i}^{\prime} \pi\left(\psi\left(a_{i}\right)\right)\right\| \leq 1 \text { for all } \psi \in \mathrm{E}_{1}(A) \text { and }\left\|\sum_{i=1}^{n} a_{i}^{\prime} \pi\left(\phi\left(a_{i}\right)\right)\right\|>1,
$$


where we have used the definitions of $S$ and $y$. In particular (taking $\psi$ of the form $\psi(x)=e_{k} x e_{k}$, where $\left(e_{k}\right)$ is an approximate unit in $A$ )

$$
\left\|\sum_{i=1}^{n} a_{i}^{\prime} \pi\left(\phi\left(a_{i}\right)\right)\right\|>\left\|\sum_{i=1}^{n} a_{i}^{\prime} \pi\left(a_{i}\right)\right\| .
$$

This contradicts assumption (ii).

It remains to prove that (iii) $\Rightarrow$ (ii). If (ii) does not hold, then there exist a (nondegenerate) representation $\pi$ of $A$ on a Hilbert space $\mathcal{H}$ and elements $a_{j} \in A$, $a_{j}^{\prime} \in \pi(A)^{\prime}(j=1, \ldots, n)$ such that (2.3) holds. Set $C=\pi(A)$ and $D=C^{*}\left(C \cup C^{\prime}\right)$, the $C^{*}$-algebra generated by $C \cup C^{\prime}$ inside $\mathrm{B}(\mathcal{H})$. By (2.3), we may choose an irreducible representation $\Theta: D \rightarrow \mathrm{B}(\mathcal{K})$ such that

$$
\left\|\Theta\left(\sum_{i=1}^{n} a_{i}^{\prime} \pi\left(\phi\left(a_{i}\right)\right)\right)\right\|>\left\|\Theta\left(\sum_{i=1}^{n} a_{i}^{\prime} \pi\left(a_{i}\right)\right)\right\| .
$$

Since $\Theta(C) \subseteq \Theta\left(C^{\prime}\right)^{\prime}$, it follows that

$$
\overline{\Theta(C)} \cap \Theta(C)^{\prime} \subseteq \Theta\left(C^{\prime}\right)^{\prime} \cap \Theta(C)^{\prime}=\Theta(D)^{\prime}=\mathbb{C} 1 ;
$$

hence $B:=\Theta(C)^{\prime}$ is a factor. Therefore the linear map $x \otimes y \stackrel{\mu}{\mapsto} x y$ from the algebraic tensor product $B \otimes \overline{\Theta(C)}$ onto the subalgebra of $\mathrm{B}(\mathcal{K})$ generated by $B \cup$ $\overline{\Theta(C)}$ is an isomorphism [11, 5.5.4]. Let $J=\operatorname{ker} \pi$. Since $A / J \cong \pi(A)=C$, we have injections

$$
B \otimes A / J \rightarrow B \otimes C \rightarrow B \otimes \overline{\Theta(C)} \stackrel{\mu}{\rightarrow} \mathrm{B}(\mathcal{K}), \quad b \otimes \dot{a} \mapsto b \Theta(\pi(a)),
$$

which can be used to define a $C^{*}$-norm on $B \otimes A / J$. Put $b_{i}=\Theta\left(a_{i}^{\prime}\right)$. Since by assumption of (iii), $\phi$ preserves ideals, $\phi$ induces a map $\phi_{J}$ on $A / J$ (such that $\phi_{J}(a+J)=\phi(a)+J$ for all $\left.a \in A\right)$. Then (2.4) says that

$$
\left\|\sum_{i=1}^{n} b_{i} \otimes \phi_{J}\left(a_{i}+J\right)\right\|>\left\|\sum_{i=1}^{n} b_{i} \otimes\left(a_{i}+J\right)\right\| .
$$

From $a_{i}^{\prime} \in \pi(A)^{\prime}=C^{\prime}$ we have $b_{i} \in \Theta\left(C^{\prime}\right) \subseteq \Theta(C)^{\prime}=B$, and by (2.5) the map $1 \otimes \phi_{J}$ on $B \otimes A / J$ is not contractive. This contradicts assumption (iii).

Now we derive some consequences of Theorem 2.1

Corollary 2.2. If the range of $\phi \in \mathrm{ICB}_{1}(A)$ is contained in a nuclear $C^{*}$-subalgebra $C$ of $A$, then $\phi \in{\overline{\mathrm{E}_{1}(A)}}^{\mathrm{p} \text {.n. }}$. In particular, if $A$ is nuclear, $\operatorname{ICB}_{1}(A)={\overline{\mathrm{E}_{1}(A)}}^{\mathrm{p} . \mathrm{n} \text {. }}$.

Proof. Suppose that $\phi \in \mathrm{ICB}_{1}(A) \backslash{\overline{\mathrm{E}_{1}(A)}}^{\mathrm{p} . \mathrm{n}}$. Then by Theorem 2.1 there exist an ideal $J$ in $A$, a $C^{*}$-algebra $B$, a $C^{*}$-norm on $B \otimes A / J$ and elements $a_{1}, \ldots, a_{n}$ in $A$ and $b_{1}, \ldots, b_{n}$ in $B$ such that (2.5) holds. Since quotients of nuclear $C^{*}$-algebras are nuclear (see [5, 9.4.4] or 21, XV. 3.4]), $C /(C \cap J)$ is nuclear; hence the $C^{*}$-tensor norm on $B \otimes C /(C \cap J)$ that comes from the injection $B \otimes C /(C \cap J) \rightarrow B \otimes A / J$ (where $B \otimes A / J$ carries the norm for which (2.5) holds) must coincide with the minimal $C^{*}$-tensor norm on $B \otimes C /(C \cap J)$. Therefore (2.5) implies that

$$
\left\|\sum_{i=1}^{n} b_{i} \otimes\left(\phi\left(a_{i}\right)+(C \cap J)\right)\right\|_{B \otimes C /(C \cap J)}>\left\|\sum_{i=1}^{n} b_{i} \otimes\left(a_{i}+J\right)\right\|_{B \otimes A / J}^{\min } .
$$


However, the inclusion $C /(C \cap J) \rightarrow A / J$ induces an (isometric) *-monomorphism $B \stackrel{\min }{\otimes} C /(C \cap J) \rightarrow B \stackrel{\min }{\otimes} A / J$; hence the last inequality can be rewritten as

$$
\left\|\sum_{i=1}^{n} b_{i} \otimes \phi_{J}\left(a_{i}+J\right)\right\|_{B \otimes A / J}>\left\|\sum_{i=1}^{n} b_{i} \otimes\left(a_{i}+J\right)\right\|_{B \otimes A / J} \min .
$$

But this contradicts the well-known fact that $1 \stackrel{\min }{\otimes} \phi_{J}$ is a complete contraction since $\phi_{J}$ is.

Recall that a (completely bounded) map $\phi: A \rightarrow B$ between $C^{*}$-algebras is called decomposable if it can be written as a linear combination of completely positive maps. Equivalently, $\phi$ is decomposable if and only if there exist completely positive maps $\psi_{j}: A \rightarrow B(j=1,2)$ such that the map

$$
A \rightarrow \mathrm{M}_{2}(B), \quad a \mapsto\left[\begin{array}{ll}
\psi_{1}(a) & \phi(a) \\
\phi\left(a^{*}\right)^{*} & \psi_{2}(a)
\end{array}\right]
$$

is completely positive. The infimum of all $\max \left\{\left\|\psi_{1}\right\|_{\mathrm{cb}},\left\|\psi_{2}\right\|_{\mathrm{cb}}\right\}$, where $\psi_{1}$ and $\psi_{2}$ range over all completely positive maps from $A$ into $B$ such that (2.6) is completely positive, is a norm, denoted by $\|\phi\|_{\text {dec }}$. For more, see [9] or [8], [18].

Corollary 2.3. For each ideal $J$ in $A$ denote by $\kappa_{J}$ the canonical embedding of $A / J$ into its second dual $(A / J)^{\sharp \sharp}=A^{\sharp \sharp} / J^{\sharp \sharp}$ (and write $\kappa=\kappa_{0}$ if $J=0$ ).

(i) If $\phi \in{\overline{\mathrm{E}_{1}(A)}}^{\mathrm{p} . \mathrm{n} .}$, then $\kappa_{J} \phi$ is decomposable with $\left\|\kappa_{J} \phi_{J}\right\|_{\mathrm{dec}} \leq 1$ for all $J \in$ $\mathcal{I}_{\mathrm{c}}(A)$.

(ii) Conversely, if $\phi \in \operatorname{ICB}_{1}(A)$ is the pointwise limit of a net of finite rank maps $\phi_{k} \in \mathrm{ICB}_{1}(A)$ such that $\left\|\kappa \phi_{k}\right\|_{\mathrm{dec}} \leq 1$, then $\phi \in{\overline{\mathrm{E}_{1}(A)}}^{\mathrm{p} . \mathrm{n} \text {. }}$. In particular, if $A$ is simple, all finite rank maps $\phi$ with $\|\phi\|_{\text {dec }} \leq 1$ are in ${\overline{\mathrm{E}_{1}(A)}}^{\mathrm{p} . \mathrm{n} \text {. }}$.

Proof. (i) By Theorem 2.1. for each $J \in \mathcal{I}_{\mathrm{c}}(A)$ and each $C^{*}$-algebra $B$ the map $1 \otimes \phi_{J}$ on $B \stackrel{\max }{\otimes} A / J$ is (completely) contractive. Hence it follows by Kirchberg's Theorem [18, 14.1] that $\kappa_{J} \phi_{J}$ is decomposable with $\left\|\kappa_{J} \phi_{J}\right\|_{\text {dec }} \leq 1$.

(ii) If $\phi \notin{\overline{\mathrm{E}_{1}(A)}}^{\text {p.n. }}$, then by Theorem 2.1 there exist $J \in \mathcal{I}_{\mathrm{c}}(A)$, a $C^{*}$-algebra $B$ and elements $a_{1}, \ldots, a_{n}$ in $A$ and $b_{1}, \ldots, b_{n}$ in $B$ satisfying (2.5), which implies that

$$
\left\|\sum_{i=1}^{n} b_{i} \otimes \phi_{J}\left(a_{i}+J\right)\right\|_{B \otimes A / J}>\left\|\sum_{i=1}^{n} b_{i} \otimes\left(a_{i}+J\right)\right\|_{B \otimes A / J} \min .
$$

Thus, for sufficiently large $k$ we have

$$
\left\|\sum_{i=1}^{n} b_{i} \otimes \phi_{k J}\left(a_{i}+J\right)\right\|_{B \otimes A / J}>\left\|\sum_{i=1}^{n} b_{i} \otimes\left(a_{i}+J\right)\right\|_{B \otimes A / J},
$$

since $\phi_{k J}\left(a_{i}+J\right)=\phi_{k}\left(a_{i}\right)+J$ converge to $\phi\left(a_{i}\right)+J=\phi_{J}\left(a_{i}+J\right)$. However, since $\left\|\kappa \phi_{k}\right\|_{\text {dec }} \leq 1,1 \otimes \phi_{k}$ is a contraction on $B \stackrel{\max }{\otimes} A$ by [18, 14.1] and since $\phi_{k}$ preserves ideals, this induces a contraction $1 \otimes \phi_{k J}$ on $\left(B \max ^{\max } A\right) /(B \otimes \max J) \cong B \otimes(A / J)$. Since $\phi_{k J}$ is of finite rank, this implies that $1 \otimes \phi_{k J}$ must be contractive as a map from $B \stackrel{\min }{\otimes} A / J$ to $B \stackrel{\max }{\otimes} A / J$ (see [18, 11.10] or [10]), contradicting (2.7).

Recall that a $C^{*}$-algebra $A$ has the weak expectation property (WEP) if the inclusion $\kappa$ of $A$ into its second dual $A^{\sharp \sharp}$ has a completely positive and completely 
contractive factorization through some $\mathrm{B}(\mathcal{H})$ [18, 15.1]. An operator space $X \subseteq$ $\mathrm{B}(\mathcal{H})$ is 1-exact if and only if for every $C^{*}$-algebra $C$ the map $i \otimes 1_{C}$, where $i: X \rightarrow$ $\mathrm{B}(\mathcal{H})$ is the inclusion, extends to a contraction $X \stackrel{\min }{\otimes} C \rightarrow \mathrm{B}(\mathcal{H}) \stackrel{\max }{\otimes} C$ [18, 17.1].

Corollary 2.4. Suppose that $A$ is simple and has the WEP and that $X$ is a 1-exact operator subspace of $A$. Then every map $\phi \in \mathrm{CB}(A)$ such that $\kappa \phi: A \rightarrow A^{\sharp \sharp}$ is decomposable with $\|\kappa \phi\|_{\text {dec }} \leq 1$ can be approximated pointwise on $X$ by elementary complete contractions from $\mathrm{E}(A)$.

Proof. If $\phi \mid X$ cannot be approximated, then for some $n \in \mathbb{N}$ there exists $x=$ $\left(x_{1}, \ldots, x_{n}\right) \in X^{n}$ such that $y:=\left(\phi\left(x_{1}\right), \ldots, \phi\left(x_{n}\right)\right)$ is not in the norm closure of the set $S:=\left\{\left(\psi\left(x_{1}\right), \ldots, \psi\left(x_{n}\right)\right): \psi \in \mathrm{E}_{1}(A)\right\}$. As in the proof of the implications (iii) $\Rightarrow\left(\right.$ ii) $\Rightarrow\left(\right.$ i) in Theorem 2.1 it follows that there exists a $C^{*}$-algebra $B$, a $C^{*}$-norm on $B \otimes A$ and elements $b_{1}, \ldots, b_{n}$ in $B$ such that (2.5) holds (with $J=0$, since $A$ is simple). This implies that

$$
\left\|\sum_{i=1}^{n} b_{i} \otimes \phi\left(x_{i}\right)\right\|_{B \max ^{\max }}>\left\|\sum_{i=1}^{n} b_{i} \otimes x_{i}\right\|_{B \otimes A}^{\min A}=\left\|\sum_{i=1}^{n} b_{i} \otimes x_{i}\right\|_{B \otimes X}^{\min X} .
$$

Let $\mathbb{F}$ be a free group such that there exists a $*$-epimorphism from the group $C^{*}$ algebra $C^{*}(\mathbb{F})$ onto $B$ and denote by $\iota$ the inclusion of $X$ into $A$. Since $A$ has the WEP, $C^{*}(\mathbb{F}) \stackrel{\max }{\otimes} A=C^{*}(\mathbb{F}) \stackrel{\min }{\otimes} A[18,15.5]$. Since $X$ is 1-exact, this implies that $1 \otimes \iota: B \stackrel{\min }{\otimes} X \rightarrow B \stackrel{\max }{\otimes} A$ is a (well-defined) contraction (see the proof of the implication (i) $\Rightarrow$ (ii) in Theorem 17.1 of [18 if necessary); hence (2.8) can be rewritten as

$$
\left\|\sum_{i=1}^{n} b_{i} \otimes \phi\left(x_{i}\right)\right\|_{B \otimes A}^{\max }>\left\|\sum_{i=1}^{n} b_{i} \otimes x_{i}\right\|_{B \max ^{\max } A}
$$

But $1 \otimes \phi$ is contractive on $B \stackrel{\max }{\otimes} A$ since $\|\kappa \phi\|_{\text {dec }} \leq 1$ [18, 14.1], a contradiction.

Problem. Characterize $C^{*}$-algebras with $\operatorname{ICB}_{1}(A)={\overline{\mathrm{E}_{1}(A)}}^{\text {p.n. }}$. Are they necessarily nuclear?

We have a partial answer to the above question. An operator space (in particular a $C^{*}$-algebra) $X$ is called locally reflexive if for each finite dimensional operator space $F$ the equality $(X \stackrel{\min }{\otimes} F)^{\sharp \sharp}=X^{\sharp \sharp} \stackrel{\min }{\otimes} F$ holds (completely) isometrically [8], [18.

Proposition 2.5. Suppose that $A$ is simple and locally reflexive. Then the equality $\mathrm{CB}_{1}(A)={\overline{\mathrm{E}_{1}(A)}}^{\text {p.n. }}$ implies that $A$ is nuclear.

Proof. We may assume that $A$ is infinite dimensional, so that $A$ contains an element with infinite spectrum [11,4.6.14]. Using a sequence of continuous functions with disjoint supports, we can construct for each $n \in \mathbb{N}$ complete contractions $\iota: \ell_{\infty}(n) \rightarrow A$ and $E: A \rightarrow \ell_{\infty}(n)$ such that $E \iota=1$ (see [9, 2.7]). To prove that the $\mathrm{W}^{*}$-envelope $\bar{A}=A^{\sharp \sharp}$ of $A$ is injective (hence $A$ is nuclear), it suffices to show that there exists a constant $c$ such that $\|\theta\|_{\text {dec }} \leq c$ for all complete contractions $\theta: \ell_{\infty}(n) \rightarrow \bar{A}$ and all $n$ [9, 2.1]. Let $n$ be fixed.

Since $A$ is locally reflexive there exists a net $\left(\theta_{k}\right)$ of complete contractions from $\ell_{\infty}(n)$ to $A$ such that the net $\left(\kappa \theta_{k}\right)$ converges to $\theta$ in the point weak* topology, where $\kappa: A \rightarrow \bar{A}$ is the canonical inclusion. Since ${\overline{\mathrm{E}_{1}(A)}}^{\text {p.n. }}=\mathrm{CB}_{1}(A)$ by assumption, for 
each $k$ there exists a net $\left(\phi_{k j}\right)_{j}$ in $\mathrm{E}_{1}(A)$ converging pointwise to $\theta_{k} E$. Since $A$ is simple (hence primitive), the natural map $A \stackrel{h}{\otimes} A \rightarrow \mathrm{CB}(A)$ is completely isometric [1. 5.4.13]; hence we have the completely isometric inclusion $\mathrm{E}(A) \subseteq A \stackrel{h}{\otimes} A$, and it follows that each $\phi_{k j} \in \mathrm{E}_{1}(A)$ is decomposable with $\left\|\phi_{k j}\right\|_{\text {dec }}=\left\|\phi_{k j}\right\|_{\mathrm{cb}}$. (To see that every $\phi \in \mathrm{E}(A)$ is decomposable, note that $\phi=a^{*} x b$, where $a$ and $b$ are fixed columns with the entries in $A$, and take in (2.6) for $\psi_{1}$ and $\psi_{2}$ the maps defined by $\psi_{1}(x)=a^{*} x a$ and $\psi_{2}(x)=b^{*} x b$.) Thus $\phi_{k j}=\sum_{\ell=0}^{3} i^{\ell} \phi_{k j \ell}$, where $\phi_{k j \ell}$ are elementary completely positive complete contractions on $A$ (and $i=\sqrt{-1}$ ). If for fixed $k$ and $\ell$ we let $\psi_{k \ell}$ be the point weak* limit of a suitable subnet of $\left(\kappa \phi_{k j \ell}\right)_{j}$, the maps $\psi_{k \ell}: A \rightarrow \bar{A}$ are completely positive and completely contractive and $\sum_{\ell=0}^{3} i^{\ell} \psi_{k \ell}=\kappa \theta_{k} E$. This shows that $\kappa \theta_{k} E$ is decomposable with $\left\|\kappa \theta_{k} E\right\|_{\text {dec }} \leq 4$. Since $\iota$ is completely positive, it follows that $\kappa \theta_{k}\left(=\kappa \theta_{k} E \iota\right)$ is also decomposable with $\left\|\kappa \theta_{k}\right\|_{\text {dec }} \leq 4$. Since the net $\left(\kappa \theta_{k}\right)$ converges to $\theta$ in the point weak* topology, it follows that $\theta$ is decomposable with $\|\theta\|_{\text {dec }} \leq 4$.

Example 2.6. Let $A=C_{\lambda}^{*}\left(\mathbb{F}_{2}\right)$ be the reduced $C^{*}$-algebra of the free group $G=\mathbb{F}_{2}$ on two generators $g_{1}, g_{2}$ and let $\phi$ be the automorphism of $A$ that interchanges the generators: $\phi\left(L_{g_{1}}\right)=L_{g_{2}}, \phi\left(L_{g_{2}}\right)=L_{g_{1}}$, where for $g \in G$ we denote by $L_{g}$ the translation operator $\left(L_{g} \xi\right)(h)=\xi\left(g^{-1} h\right)\left(\xi \in \ell_{2}(G)\right)$. Since $A$ is simple [7], $\phi \in \mathrm{ICB}_{1}(A)$. We will show that $\phi \notin{\overline{\mathrm{E}_{1}(A)}}^{\text {p.n. }}$. The same argument then shows that the weak* continuous extension $\bar{\phi}$ of $\phi$ to the von Neumann algebra $\bar{A}=W^{*}(G)$ is not in ${\overline{\mathrm{E}_{1}(\bar{A})}}^{\text {p.n. }}$, which is a much stronger result than the classical fact that the automorphism $\bar{\phi}$ is not inner [11, 6.9.43]. Recall that the commutant $A^{\prime}$ of $A$ in $\mathrm{B}\left(\ell_{2}(G)\right)$ is the von Neumann algebra generated by the right convolvers $R_{g}(g \in G)$, where $\left(R_{g} \xi\right)(h)=\xi(h g)\left(\xi \in \ell_{2}(G)\right)$. Note that $\phi$ maps the elements $1, L_{g_{1}}, L_{g_{1}}^{*}, L_{g_{2}}$ into $1, L_{g_{2}}, L_{g_{2}}^{*}, L_{g_{1}}$ (respectively). By Theorem 2.1, to show that $\phi \notin \overline{\mathrm{E}_{1}(A)}$ p.n. it suffices to show that

$$
\left\|1+L_{g_{2}} R_{g_{2}}+L_{g_{2}}^{*} R_{g_{2}}^{*}+L_{g_{1}} R_{g_{1}}\right\|>\left\|1+L_{g_{1}} R_{g_{2}}+L_{g_{1}}^{*} R_{g_{2}}^{*}+L_{g_{2}} R_{g_{1}}\right\| .
$$

The operator on the left side of (2.9) is a sum of four unitary operators and maps the unit vector $\delta_{e}\left(e\right.$ is the unit of $G$ ) to $4 \delta_{e}$; hence the left side of (2.9) is equal to 4. Suppose (to reach a contradiction) that (2.9) does not hold. Then, given $\varepsilon>0$, there exists a unit vector $\xi=\sum \xi(g) \delta_{g}$ in $\ell_{2}(G)$ such that

$$
\left\|\left(1+L_{g_{1}} R_{g_{2}}+L_{g_{1}}^{*} R_{g_{2}}^{*}+L_{g_{2}} R_{g_{1}}\right) \xi\right\|>4-\frac{\varepsilon^{2}}{4} .
$$

On the left side of (2.10) we have a sum of four unit vectors in a Hilbert space, and it follows by an elementary argument (see e.g. [18, Exercise 20.2]) that

$$
\left\|L_{g_{1}} R_{g_{2}} \xi-\xi\right\|<\varepsilon,\left\|L_{g_{1}}^{*} R_{g_{2}}^{*} \xi-\xi\right\|<\varepsilon \text { and }\left\|L_{g_{2}} R_{g_{1}} \xi-\xi\right\|<\varepsilon .
$$

This means that

$$
\begin{aligned}
\sum_{g \in G}\left|\xi\left(g_{1}^{-1} g g_{2}\right)-\xi(g)\right|^{2}<\varepsilon^{2}, & \sum_{g \in G}\left|\xi\left(g_{1} g g_{2}^{-1}\right)-\xi(g)\right|^{2}<\varepsilon^{2} \text { and } \\
& \sum_{g \in G}\left|\xi\left(g_{2}^{-1} g g_{1}\right)-\xi(g)\right|^{2}<\varepsilon^{2} .
\end{aligned}
$$


Now we perform a similar (but not identical) computation as in [21, IVX.3.9] (or [11). For each subset $T$ of $G=\mathbb{F}_{2}$ let

$$
\mu(T)=\sum_{g \in T}|\xi(g)|^{2} .
$$

Let $S$ be the subset of $\mathbb{F}_{2}$ that contains the unit and all the reduced words $w \in \mathbb{F}_{2}$ which end with $g_{1}^{j}$ with $j \neq 0$. Note that $S \cup g_{2}^{-1} S g_{1}=\mathbb{F}_{2}$ and that the sets $S$, $g_{1}^{-1} S g_{2}$ and $g_{1} S g_{2}^{-1}$ are disjoint. Therefore

$$
\begin{gathered}
\mu(S)+\mu\left(g_{2}^{-1} S g_{1}\right) \geq 1 \text { and } \\
\mu(S)+\mu\left(g_{1}^{-1} S g_{2}\right)+\mu\left(g_{1} S g_{2}^{-1}\right) \leq 1 .
\end{gathered}
$$

Since $|\|x\|-\|y\|| \leq\|x-y\|$ for any two vectors $x, y \in \ell_{2}(S)$, we have

$$
\left|\mu\left(g_{1}^{-1} S g_{2}\right)^{1 / 2}-\mu(S)^{1 / 2}\right| \leq\left(\sum_{g \in G}\left|\xi\left(g_{1}^{-1} g g_{2}\right)-\xi(g)\right|^{2}\right)^{1 / 2}
$$

hence the first inequality in (2.11) implies that

$$
\left|\mu\left(g_{1}^{-1} S g_{2}\right)-\mu(S)\right|=\left|\mu\left(g_{1}^{-1} S g_{2}\right)^{1 / 2}-\mu(S)^{1 / 2}\right|\left|\mu\left(g_{1}^{-1} S g_{2}\right)^{1 / 2}+\mu(S)^{1 / 2}\right|<2 \varepsilon .
$$

Applying the same reasoning to the other two inequalities in (2.11) we get

$$
\left|\mu\left(g_{1}^{-1} S g_{2}\right)-\mu(S)\right|<2 \varepsilon,\left|\mu\left(g_{1} S g_{2}^{-1}\right)-\mu(S)\right|<2 \varepsilon,\left|\mu\left(g_{2}^{-1} S g_{1}\right)-\mu(S)\right|<2 \varepsilon .
$$

The last of these three inequalities together with (2.12) implies that $\mu(S)>\frac{1}{2}-\varepsilon$. On the other hand, the first two inequalities in (2.15) together with (2.13) imply that $3 \mu(S)-4 \varepsilon<1$, and hence we have $(1 / 2)-\varepsilon<\mu(S)<(1+4 \varepsilon) / 3$. This is a contradiction if $\varepsilon \leq 1 / 14$.

\section{LifTING AND EXTENSIONS}

Since it is not always easy to verify the conditions of Theorem 2.1, we will present another obstruction for approximation by elementary complete contractions. As an application, we show that the Calkin algebra does not have the ECCAP. This fact seems interesting, since Voiculescu's theorem [3, Theorem 4] (together with Paulsen's technique of $2 \times 2$ matrices) implies that each complete contraction on $\mathrm{B}(\mathcal{H})(\mathcal{H}$ separable) which annihilates the ideal $\mathrm{K}(\mathcal{H})$ of compact operators can be approximated pointwise by two-sided multiplications $x \mapsto a x b$ with $a, b \in \mathrm{B}(\mathcal{H})$ contractive.

Proposition 3.1. Let $J \in \mathcal{I}_{\mathrm{c}}(A), B:=A / J, q: A \rightarrow B$ be the quotient map, $X$ be a separable subspace of $A$ and $\psi \in{\overline{\mathrm{E}_{1}(B)}}^{\text {p.n. }}$. Then there exists a sequence $\left(\phi_{k}\right)$ in $\mathrm{E}_{1}(A)$ converging pointwise on $X$ to a complete contraction $\phi: X \rightarrow A$ such that $q \phi=\psi q \mid X$. That is, the following diagram commutes:

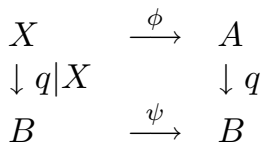

Proof. Let $\mathrm{E}_{1}^{h}(B)$ be the set of all $\psi \in \mathrm{E}(B)$ which can be represented by elements $w \in B \otimes B$ with the Haagerup norm $\|w\|_{h}<1$. Since the Haagerup norm dominates the c.b. norm, $\mathrm{E}_{1}^{h}(B) \subseteq \mathrm{E}_{1}(B)$. Note that each $\psi \in \mathrm{E}_{1}^{h}(B)$ can be lifted to an operator $\phi \in \mathrm{E}(A)$ (that is, $q \phi=\psi q$ ) with $\|\phi\|_{\mathrm{cb}}<1$. Indeed, let $\psi$ be represented 
by a tensor $w=\sum_{j=1}^{n} \dot{a}_{j} \otimes \dot{b}_{j}$, where the row matrix $\dot{a}:=\left[\dot{a}_{1}, \ldots, \dot{a}_{n}\right]$ and the column matrix $\dot{b}:=\left[\dot{b}_{1}, \ldots, \dot{b}_{n}\right]^{T}$ (with the entries in $B$ ) have norms less than 1 . Regarding $\dot{a}$ and $\dot{b}$ as elements of the $C^{*}$-algebra $\mathrm{M}_{n}(B)=\mathrm{M}_{n}(A / J)=\mathrm{M}_{n}(A) / \mathrm{M}_{n}(J)$ (by completing with zero entries), we may lift $\dot{a}$ and $\dot{b}$ to a suitable row $a$ and a column $b$, both with the entries in $A$, so that the corresponding elementary operator $\phi(x):=$ $\sum_{j=1}^{n} a_{j} x b_{j}$ on $A$ satisfies $\|\phi\|_{\mathrm{cb}} \leq\|a\|\|b\|<1$. Clearly $\phi$ is a lift of $\psi$ (that is, $q \phi=\psi q)$.

Now we claim that $\mathrm{E}_{1}(B) \subseteq{\overline{\mathrm{E}_{1}^{h}(B)}}^{\mathrm{p} . \mathrm{n}}$. Assume to the contrary that there exists a $\phi \in \mathrm{E}_{1}(B) \backslash{\overline{\mathrm{E}_{1}^{h}(B)}}^{\mathrm{p} . \mathrm{n} .}$. Observe that in the argument of the proof of the implication (ii) $\Rightarrow$ (i) in Theorem 2.1 we may replace the set $\mathrm{E}_{1}(A)$ with $\mathrm{E}_{1}^{h}(A)$ since [14, 1.1] is still applicable. Then (in our present context of the $C^{*}$-algebra $B$ instead of $A$ ) the argument shows that there exist a representation $\pi$ of $B$ and finite subsets $\left\{a_{1}, \ldots, a_{n}\right\}$ of $B$ and $\left\{a_{1}^{\prime}, \ldots, a_{n}^{\prime}\right\}$ of $\pi(B)^{\prime}$ such that (2.3) holds. But this is impossible since $\phi \in \mathrm{E}_{1}(B)$.

For a general $\psi \in{\overline{\mathrm{E}_{1}(B)}}^{\text {p.n. }}$, by the previous paragraph there exists a net $\left(\psi_{k}\right)$ in $\mathrm{E}_{1}^{h}(B)$ converging pointwise to $\psi$. Then the net of maps $\psi_{k} q \mid X$ converges to $\psi q \mid X$ pointwise on $X$. By the first paragraph of the proof for each $\psi_{k}$ there exists $\phi_{k} \in \mathrm{E}_{1}(A)$ such that $q \phi_{k}=\psi_{k} q$; hence in particular $q \phi_{k}\left|X=\psi_{k} q\right| X$. Since $X$ is separable, it follows by the proof of the Arveson lifting theorem [3] (see also [18, p. 425]) that $\psi q \mid X$ has a completely contractive lifting $\phi: X \rightarrow A$ such that $\phi$ is the pointwise limit of a sequence of operators in $\mathrm{E}_{1}(A)$ restricted to $X$.

Example 3.2. Let $\mathcal{H}$ be a separable Hilbert space, $B:=\mathrm{B}(\mathcal{H}), K:=\mathrm{K}(\mathcal{H})$ be the ideal of compact operators in $B, C:=B / K$ be the Calkin algebra and $q: B \rightarrow C$ be the quotient map. Consider a copy $B_{0}$ of $\ell_{\infty}(n)(n \geq 3)$ of infinite multiplicity inside $B$ so that $q$ maps $B_{0}$ completely isometrically onto a $C^{*}$-subalgebra $C_{0}$ of $C$. Let $E: C \rightarrow C_{0}$ be a completely contractive (completely positive) projection (which exists since $\left.C_{0} \cong \ell_{\infty}(n)\right)$. Since by [9, 3.2] $\ell_{\infty}(n)$ does not have the 1-operator (local) lifting property in the sense of [18, Section 16], by Ozawa's theorem [16], [18, 16.10] there exists a complete contraction $\psi_{0}: C_{0} \rightarrow C$ which does not have any completely contractive lifting $C_{0} \rightarrow B$. Set $\psi:=\psi_{0} E$, a complete contraction on $C$. Then there is no completely contractive lift $\phi: B_{0} \rightarrow B$ of $\psi_{0}=\psi \mid C_{0}$; otherwise $\phi\left(q \mid B_{0}\right)^{-1}: C_{0} \rightarrow B$ would be a completely contractive lift of $\psi_{0}$. It follows now from Proposition 3.1 that $\psi \notin{\overline{\mathrm{E}_{1}(C)}}^{\text {p.n. }}$.

It is not hard to prove that an ideal of a $C^{*}$-algebra with the ECCAP also has the ECCAP, but the author does not know if this property passes to quotients. Using Proposition 3.1 we can show that an extension by a nuclear ideal of a $C^{*}$-algebra having ECCAP has the ECCAP.

Proposition 3.3. Let $0 \rightarrow J \rightarrow A \stackrel{q}{\rightarrow} B \rightarrow 0$ be an exact sequence of $C^{*}$-algebras, where $A$ is separable and $J$ is nuclear. Then, if $B$ has the ECCAP, the same holds for $A$.

Proof. Let $\phi \in \operatorname{ICB}_{1}(A)$ and denote by $\psi$ the map on $B$ induced by $\phi$ so that $\psi q=q \phi$. By Proposition 3.1 there exists $\phi_{0} \in{\overline{\mathrm{E}_{1}(A)}}^{\mathrm{p} . n \text {. }}$ such that $q \phi_{0}=\psi q$. Thus $q\left(\phi-\phi_{0}\right)=0$; hence $\left(\phi-\phi_{0}\right)(A) \subseteq J$. Let $\left(e_{k}\right)_{k \in \mathbb{K}}$ be a quasicentral approximate unit in $J \subseteq A$ (see e.g. [3] or [7]). Since $e_{k} \phi(x) \in J$ for all $x \in A$ and $J$ is nuclear, by Corollary 2.2 for each $m \in \mathbb{K}$ the map $e_{m} \phi$ is in ${\overline{\mathrm{E}_{1}(A)}}^{\mathrm{p} . \mathrm{n} \text {. }}$. Thus, both $\phi_{0}$ and 
$e_{m} \phi$ are in ${\overline{\mathrm{E}_{1}(A)}}^{\text {p.n. }}$; hence the same must hold for the complete contraction

$$
\theta_{k m}(x):=\sqrt{1-e_{k}} \phi_{0}(x) \sqrt{1-e_{k}}+\sqrt{e_{k}}\left(e_{m} \phi(x)\right) \sqrt{e_{k}}(x \in A)
$$

for each $k \in \mathbb{K}$. Since $\sqrt{e_{k}} \in J$ and $\left(e_{m}\right)$ is an approximate unit for $J$,

$$
\theta_{k}(x):=\lim _{m \rightarrow \infty} \theta_{k m}(x)=\sqrt{1-e_{k}} \phi_{0}(x) \sqrt{1-e_{k}}+\sqrt{e_{k}} \phi(x) \sqrt{e_{k}} \text { for each } x \in A,
$$

so that $\theta_{k} \in{\overline{\mathrm{E}_{1}(A)}}^{\mathrm{p} \text {.n. }}$. But we can write

$$
\theta_{k}(x)=\left(\sqrt{1-e_{k}} \phi(x) \sqrt{1-e_{k}}+\sqrt{e_{k}} \phi(x) \sqrt{e_{k}}\right)+\sqrt{1-e_{k}}\left(\phi_{0}(x)-\phi(x)\right) \sqrt{1-e_{k}}
$$

and, since $\left(\phi_{0}-\phi\right)(x) \in J$ and the approximate unit $\left(e_{k}\right)$ is quasicentral in $A$, it follows that $\lim _{k \rightarrow \infty} \theta_{k}(x)=\phi(x)$. Thus $\phi \in{\overline{\mathrm{E}_{1}(A)}}^{\mathrm{p} \text {.n. }}$.

\section{ACKNOWLEDGMENT}

The author is grateful to Richard Timoney for his suggestions.

\section{REFERENCES}

[1] P. Ara and M. Mathieu, Local multipliers of $C^{*}$-algebras, Springer Monographs in Math., Springer-Verlag, London, 2003. MR1940428 (2004b:46071)

[2] R. J. Archbold, D. W. B. Somerset and R. M. Timoney, On the central Haagerup tensor product and completely bounded mappings of a $C^{*}$-algebra, J. Funct. Anal. 226 (2005), 406428. MR2160102 (2006d:46066)

[3] W. B. Arveson, Notes on extensions of $C^{*}$-algebras, Duke Math. J. 44 (1977), 329-355. MR0438137 (55:11056)

[4] D. P. Blecher and C. Le Merdy, Operator algebras and their modules-an operator space approach, London Math. Soc. Monographs, New Series, 30, Clarendon Press, Oxford, 2004. MR.2111973 (2006a:46070)

[5] N. P. Brown and N. Ozawa, $C^{*}$-algebras and finite dimensional approximations, Graduate Studies in Math., 88, Amer. Math. Soc., Providence, RI, 2008. MR2391387

[6] A. Chatterjee and R. R. Smith, The central Haagerup tensor product and maps between von Neumann algebras, J. Funct. Anal. 112 (1993), 97-120. MR.1207938 (94c:46120)

[7] K. Davidson, $C^{*}$-algebras by example, Fields Institute Monographs 6, Amer. Math. Soc., Providence, RI, 1996. MR1402012 (97i:46095)

[8] E. G. Effros and Z.-J. Ruan, Operator spaces, London Math. Soc. Monographs, New Series, 23, Oxford University Press, Oxford, 2000. MR.1793753 (2002a:46082)

[9] U. Haagerup, Injectivity and decomposition of completely bounded maps, Lecture Notes in Math. 1132, Springer-Verlag, Berlin, 1985, pp. 170-222. MR799569 (87i:46133)

[10] M. Junge and C. Le Merdy, Factorization through matrix spaces for finite rank operators between $C^{*}$-algebras, Duke Math. J. 100 (1999), 299-319. MR.1722956 (2000m:46121)

[11] R. V. Kadison and J. R. Ringrose, Fundamentals of the theory of operator algebras, Vols. 1, 2, Academic Press, London, 1983, 1986. MR0719020 (85j:46099) MR0859186 (88d:46106)

[12] B. Magajna, A transitivity theorem for algebras of elementary operators, Proc. Amer. Math. Soc. 118 (1993), 119-127. MR1158004 (93f:46092)

[13] B. Magajna, A transitivity problem for completely bounded mappings, Houston J. Math. 23 (1997), 109-120. MR.1688839 (2000d:46073)

[14] B. Magajna, $C^{*}$-convex sets and completely bounded bimodule homomorphisms, Proc. Roy. Soc. Edinburgh 130A (2000), 375-387. MR1750836 (2001g:46134)

[15] B. Magajna, Uniform approximation by elementary operators, http://www.fmf. uni-lj.si/ ${ }^{\sim}$ magajna/Publications/Uniform.pdf, to appear in Proc. Edinburgh Math. Soc.

[16] N. Ozawa, On the lifting property for universal $C^{*}$-algebras of operator spaces, J. Operator Theory 46 (2001), 579-591. MR1897155 (2003c:46073)

[17] V. I. Paulsen, Completely bounded maps and operator algebras, Cambridge Studies in Advanced Mathematics 78, Cambridge University Press, Cambridge, 2002. MR1976867 (2004c:46118)

[18] G. Pisier, Introduction to operator space theory, London Math. Soc. Lecture Note Series, 294, Cambridge Univ. Press, Cambridge, 2003. MR2006539 (2004k:46097) 
[19] R. R. Smith. Completely bounded module maps and the Haagerup tensor product, J. Funct. Anal. 102 (1991), 156-175. MR.1138841 (93a:46115)

[20] D. W. B. Somerset, The central Haagerup tensor product of a $C^{*}$-algebra, J. Operator Theory 39 (1998), 113-121. MR1610306 (99e:46073)

[21] M. Takesaki, Theory of operator algebras. I, III, Springer-Verlag, New York, 1979, 2001. MR548728 (81e:46038) MR.1943007 (2004g:46080)

[22] S. Wassermann, Exact $C^{*}$-algebras and related topics, Lecture Notes Series 19, Seoul Nat. Univ., 1994. MR1271145 (95b:46081)

Department of Mathematics, University of Ljubljana, Jadranska 21, Ljubljana 1000 , SLOVENIA

E-mail address: Bojan.Magajna@fmf.uni-lj.si 